\title{
Performance Enhancement of VNSIP approach, using MCAC algorithm
}

\author{
I. MOURTAJI ${ }^{1}$, M. BOUHORMA ${ }^{2}$, M. BENAHMED ${ }^{3}$, A. BOUHDIR ${ }^{4}$ \\ ${ }^{1234}$ LIST laboratory, FST of Tangier \\ E-mail: ${ }^{1}$ imourtaji@gmail.com, ${ }^{2}$ bouhorma@gmail.com, ${ }^{3}$ med.benahmed@gmail.com, \\ ${ }^{4}$ hakim.anouar@gmail.com
}

\begin{abstract}
Ad Hoc Networks provide a real opportunity to design flexible networks, very simple to deploy. However they remain a particular computation environment, characterized by the deficiency of pre-existed and centralized infrastructure. In the other hand, SIP protocol, which knows a huge booming in internet networks, requires centralized entities, like proxy server, registrar server and location service; consequently SIP is not adapted to Ad Hoc networks. We have presented in a new technique VNSIP (Virtual Network for Session Initiation Protocol) to fix the problem related to constraints of SIP deployment in MANET (Mobile Ad Hoc Networks). In this paper we use a new algorithm which we have called MCAC (MANET Call Admission Control) to improve VNSIP Performances.
\end{abstract}

Keywords: Ad hoc, SIP, MCAC, Evaluation of performance.

\section{INTRODUCTION}

VNSIP [13] allows decentralization of SIP proxies, specially registrar, proxy and location servers $[1,2,10]$, by integrating those server functionalities in each MANET node. VNSIP node contains a supervisor module VNA (Virtual Network Algorithm), it is responsible of activating and deactivating server functionalities according to the position of the node in the MANET [3][7]. To achieve evaluation of performances of VNSIP, we have compared our solution to the TCA approach [9], which is consider as a solution giving a very interesting result to fix the problem of SIP adaptation to Ad Hoc Networks. Simulations of both approaches has shown that our solution guarantees better results than TCA in terms of setting time and failure rates of SIP sessions. These good results were obtained thanks to VNA, the algorithm used to construct the VNSIP topology, and thanks to the mechanism of replication of SIP messages. On the other side, this mechanism has its own disadvantage, which involves that our solution proposes lower results than TCA in term of bandwidth consumption. to improve those results we propose a new technique, MCAC (MANET Call Admission Control), deployed on service level, this application will guarantee that a limited number of calls is controlled in the MANET and QoS is ensured. When the threshold of calls is reached, MANET proceeds interdiction of new calls.

This paper is organized as follows. In the first section we'll give an overview of VNSIP solution. In the second section we'll present MCAC algorithm. Afterward in the next section, we'll present implementation of MCAC to VNSIP approach, and in the last section we'll evaluate new performances of VNSIP. In the end, we'll achieve this paper with a conclusion, with future works and perspectives.

\section{VNSIP OVERVIEW}

VNSIP solution [13] tries to define a dynamic virtual network inside the MANET, to be exploited by nodes, to adequately choose which one will be in charge to execute SIP server tasks. VNA (Virtual Network Algorithm) will be the entity in charge to activate or deactivate SIP server functionalities in each MANET node. A VNSIP node (see figure 1) consists of several entities, when interacting between them; they allow communication in MANET using SIP protocol. 


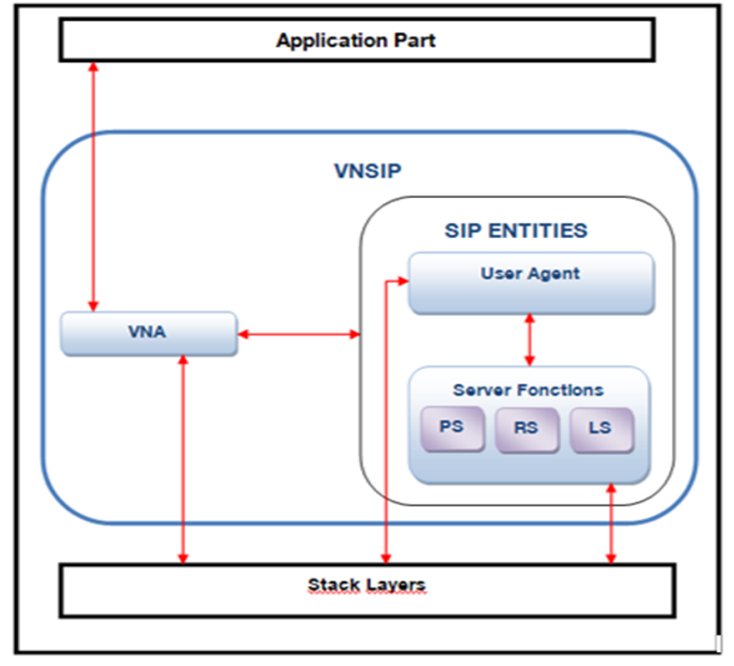

Fig. 1. VNSIP Node Architecture

\subsection{Virtual Network Algorithm (VNA)}

VNA starts by the construction of neighbor tables. VNA defines a flag "VN_membership_flag" which shows if a node belongs to the VN (Virtual Nework) or not. When executing VNA, the VN will include all nodes having VN_membership_flag=1. VNA is characterized by two conditions:

- Condition1: if a node doesn't belong to the $\mathrm{VN}$ and the number of its neighbors which belong to the $\mathrm{VN}$ is lower than the number of its neighbors which don't belong to the VN then the VN_membership_flag of this node is set to 1 .

- Condition 2: if a node belongs to the $\mathrm{VN}$ and the number of its neighbors which belong to the $\mathrm{VN}$ is higher than the number of its neighbors which don't belong to the $\mathrm{VN}$ then the VN_membership_flag of this node is set to 0 .

To complete the construction of a connected VN, we define Gateway nodes to ensure connections between all nodes belonging to the VN. When finishing the selection of gateway nodes the construction of the $\mathrm{VN}$ is completed (see figure 2).

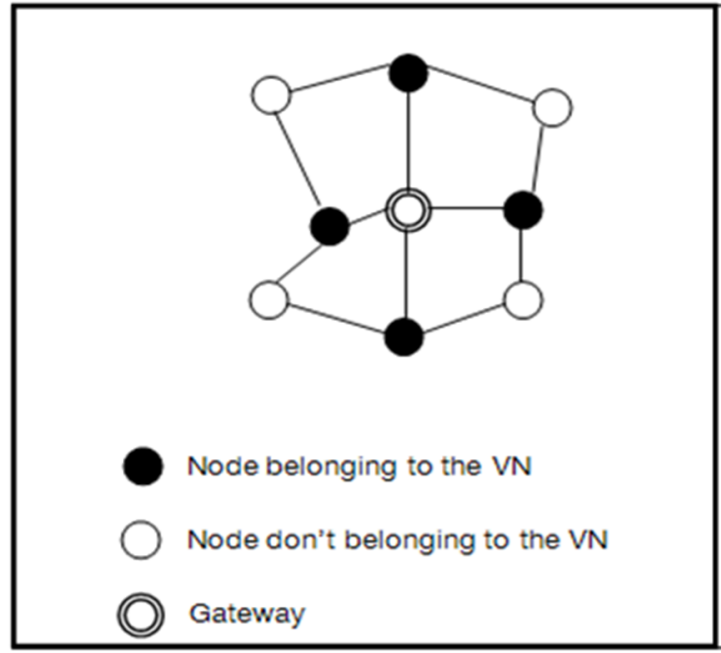

Fig. 2. VN construction

\subsection{Interaction between VNA and SIP entities}

As it shown in figure 2, when finishing construction of the $\mathrm{VN}$, three kinds of nodes are defined:

- If a node belongs to the VN, then it plays SIP User Agent role and all its SIP server's functionalities (Proxy server (PS), Registrar (RG) and location service (LS)) [6] are activated,

- If a node is a gateway, then it plays SIP User Agent role and $\mathrm{PS}$ role. $\mathrm{RG}$ and $\mathrm{LS}$ are deactivated.

- If a node doesn't belong to the $\mathrm{VN}$, then it plays only SIP User Agent role and its SIP server's functionalities are deactivated.

3

\section{MCAC: MANET CALL ADMISSION CONTROL}

The purpose of this chapter is to describe our solution, MCAC (MANET Call Admission Control), This feature allows to limit VoIP calls in ad hoc networks. The need of MCAC for VoIP 
I. mourtaji et al. / International Journal of Computer Networks and Communications Security, 1 (3), August 2013

communications is mainly because the bandwidth of the access network of a MANET is not sufficient to accept all communications simultaneously. The purpose of the MCAC solution is to allow the establishment of a number of communications in line with the available bandwidth on the network access of a MANET. Its vocation is to reject the establishment of communications when the bandwidth is no longer sufficient and thus not disrupt communications already established.

The MANET is related to a set of Getaways which are responsible to provide connectivity of MANET with the internet network (figure3). On each Getaway we integrate MCAC Application Server, which contains MCAC process and MCAC database. Each Getaway, via its Application Server, defines an MCAC group, and associates this MCAC group to a list of MANET nodes and a number of simultaneous calls [4][5]. When a part of the MANET, subscribes to a MCAC group, a call between two nodes of this group will be recognized by the MCAC process.

To create MCAC groups, Getaways (Application Server) determines MANET nodes with which it is directly linked, and automatically associates them to MCAC group.

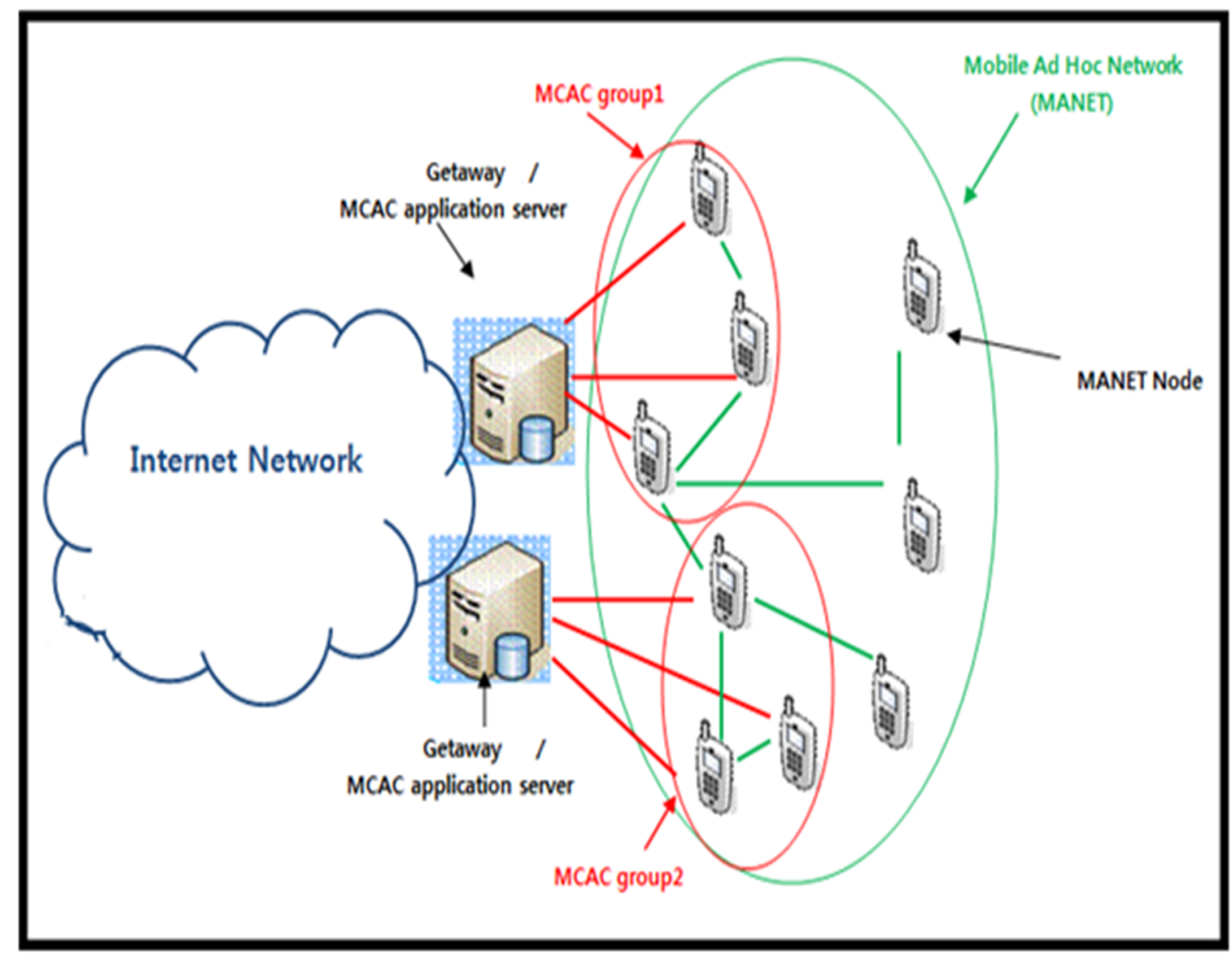

Fig. 3. Architecture of MANET connectivity to internet

Each node can be associated with one and only one MCAC group (Figure 4). When a network node is associated with a MCAC group, all calls, incoming or outgoing, concerning this node are supervised by the MCAC algorithm. If the threshold of the MCAC group associated with this line is reached, MCAC algorithm rejects any new calls, by sending the specific SIP error code « 503 service unavailable », when receiving this error, the node is assumed to play a congestion tone. 


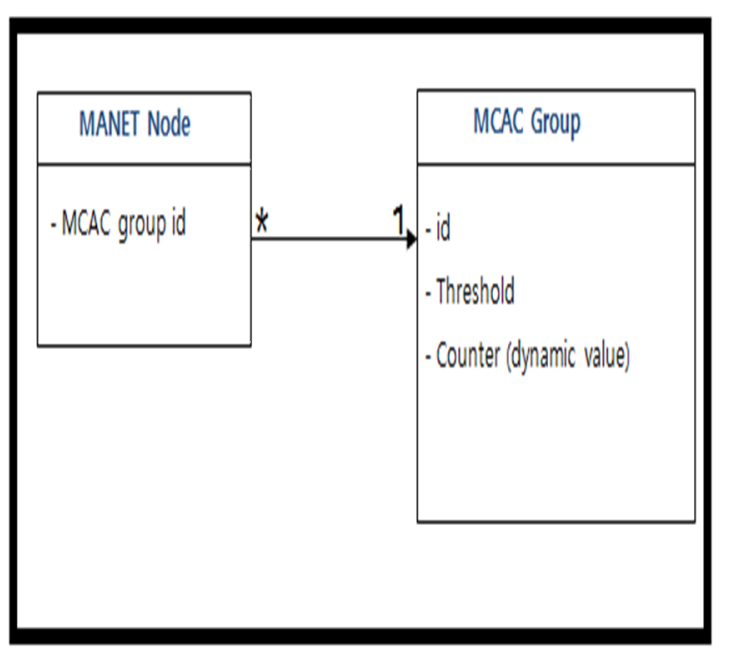

Fig. 4. MCAC data model

When a Node A decides to communicate with a node B, several scenarios to increment and decrement the MCAC counter are defined, according to if $\mathrm{A}$ and $\mathrm{B}$ belong to an MCAC group or not. The table 1 shows different possible use cases of increasing and decreasing the MCAC counter.

\section{Table 1: MCAC use cases}

\begin{tabular}{|c|c|c|}
\hline Use case & $\begin{array}{l}\text { MCAC Group } \\
\text { counter of } A\end{array}$ & $\begin{array}{l}\text { MCAC Group } \\
\text { counter of } B\end{array}$ \\
\hline $\begin{array}{l}\text { A calls B. B } \\
\text { belongs to the } \\
\text { same MCAC } \\
\text { Group than A }\end{array}$ & $\begin{array}{l}+1 \text { at set-up time } \\
-1 \text { at release time }\end{array}$ & $\begin{array}{l}-1 \text { at set-up time } \\
-1 \text { at release time }\end{array}$ \\
\hline $\begin{array}{l}\text { A calls B. B } \\
\text { belongs to another } \\
\text { MCAC Group } \\
\text { than A }\end{array}$ & $\begin{array}{l}+1 \text { at set-up time } \\
-1 \text { at release time }\end{array}$ & $\begin{array}{l}+1 \text { at set-up time } \\
-1 \text { at release time }\end{array}$ \\
\hline $\begin{array}{l}\text { A calls B. B has } \\
\text { no CAC Group } \\
\text { associated }\end{array}$ & $\begin{array}{l}+1 \text { at set-up time } \\
-1 \text { at release time }\end{array}$ & Not applicable \\
\hline $\begin{array}{l}\text { A calls B. A has } \\
\text { no CAC Group } \\
\text { associated }\end{array}$ & Not applicable & $\begin{array}{l}+1 \text { at set-up time } \\
-1 \text { at release time }\end{array}$ \\
\hline
\end{tabular}

If a node of the MANET, associated to a MCAC group, decides to perform a call to another node of the MANET, the MANET routing protocol must ask authorization from MCAC algorithm, before routing the call to destination node. The figure 5 shows an example of Scenario, in this case A and B are belonging to the same MCAC group.

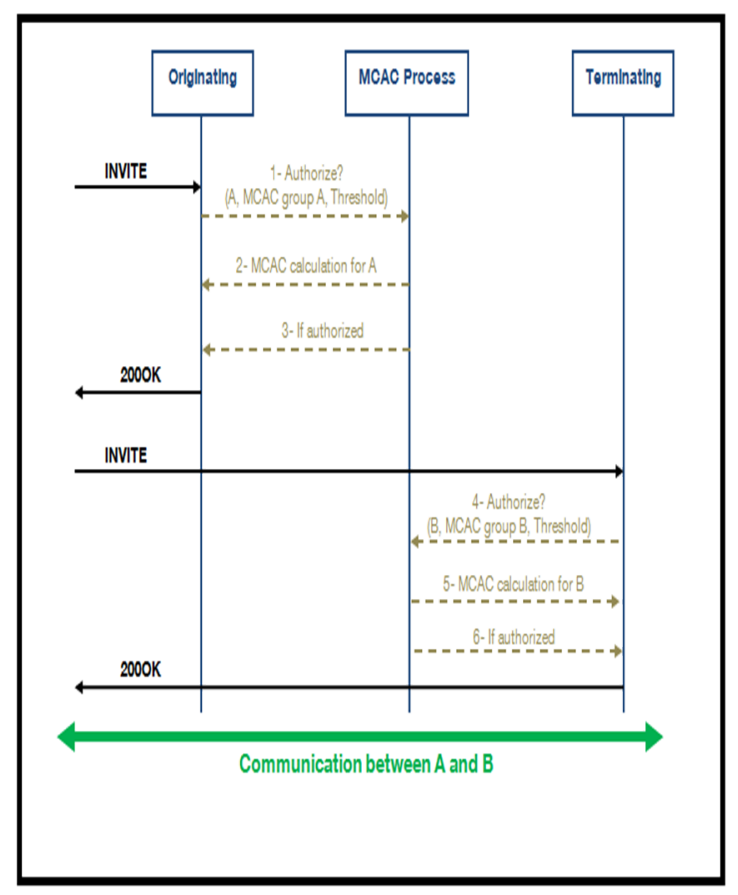

Fig. 5. Example of scenario of communication between two Nodes controlled by MCAC process

\section{APPLICATION OF MCAC ALGORITHM TO VNSIP APPROACH}

As we have mentioned in the introduction, VNSIP approach [13], comparing to TCA approach performs good results concerning time of session establishment and failure rates, but in the other side it's suffering from a high consumption of bandwidth because of the mechanism of replication of SIP messages. To resolve this problem we deploy MCAC algorithm on VNSIP architecture (see figure 6). MCAC will control SIP communication established by VNSIP, which involves controlling bandwidth consumption to be in line with the MANET capacity. 
I. mourtaji et al. / International Journal of Computer Networks and Communications Security, 1 (3), August 2013

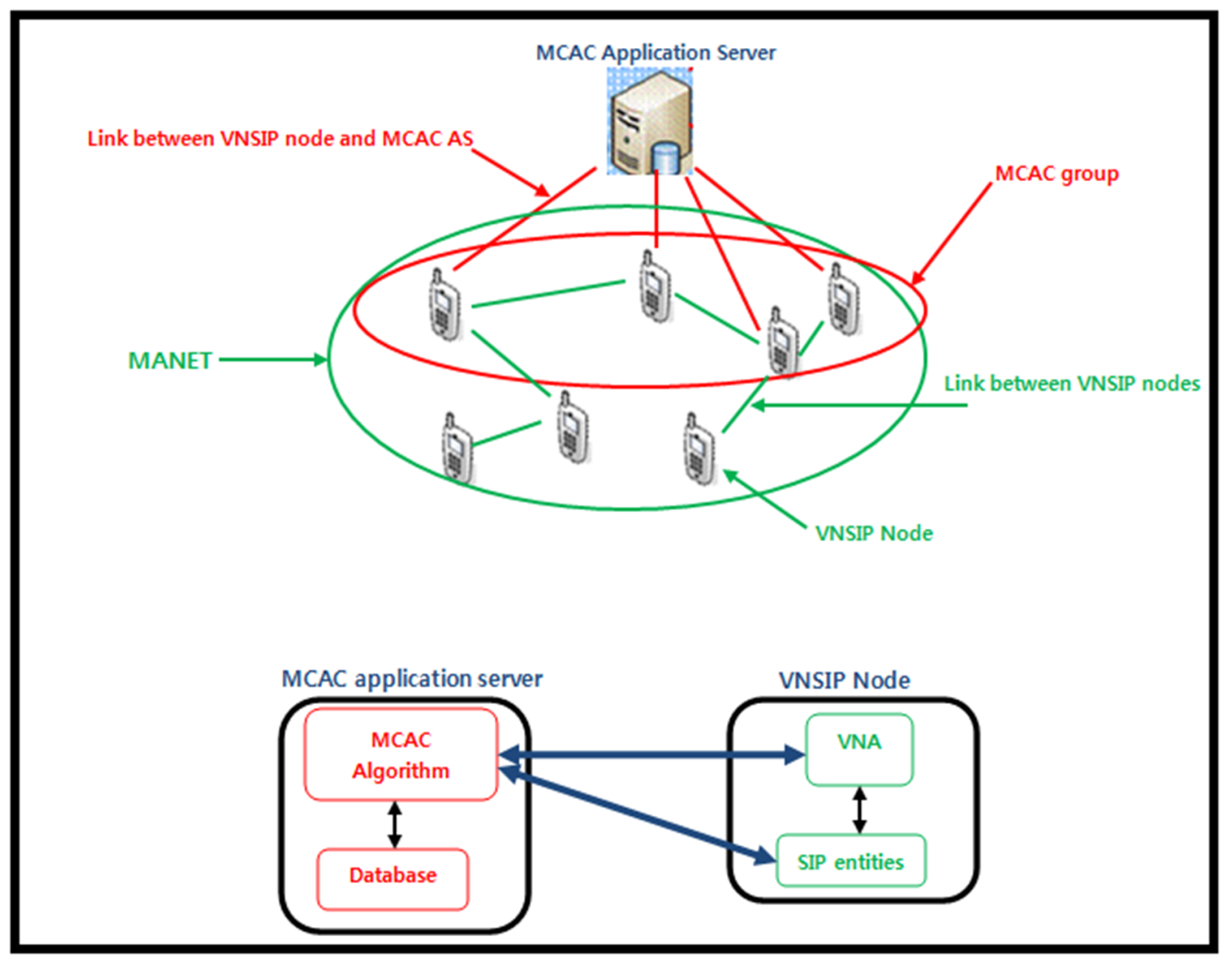

Fig. 6. Deployment of MCAC on VNSIP architecture

When a VNSIP node, which belongs to the MCAC group, decides to initiate a call with another VNSIP node, the VNA (Virtual Network Algorit$\mathrm{hm}$ ) of this node contacts the MCAC algorithm (see figure 6), before redirecting the call to the PS (Proxy server) of the destination node, to ask if it's authorized to perform the call. If the counter of simultaneous calls is still lower than the threshold defined in MCAC database, then the call will be correctly redirected to destination and the counter will be incremented. If not, then the MCAC algorithm will send a specific SIP error.

When the destination receives the call request, two cases are treated. Firstly, if the destination doesn't belong to the MCAC group then the MCAC algorithm will not be invoked and the call will be normally treated. Secondly, if the destination belongs to the MCAC group, then its VNA will also ask authorization from MCAC algorithm to treat the call. If the threshold is not yet reached then the call will be correctly treated. If not, the MCAC algorithm will send the SIP error. The figure 7 illustrates an example of a call establishment scenario between two VNSIP nodes which belong to the MCAC group.

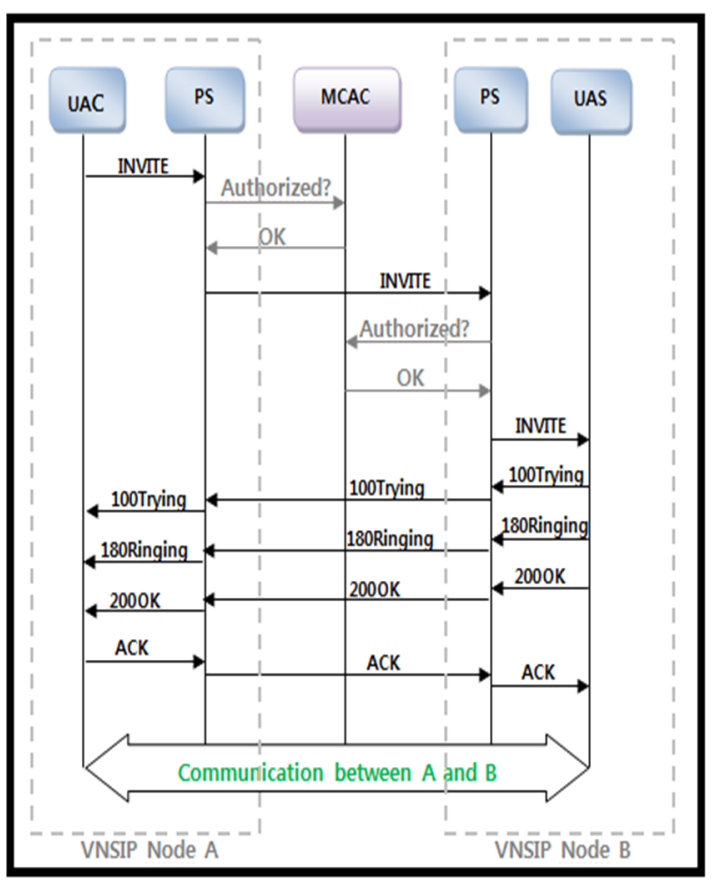

Figure 7 call flow between two nodes under MCAC control 


\section{SIMULATIONS AND EVALUATION OF PERFORMANCES}

Simulations scenarios were achieved using the network simulator NS2 [8]. The simulation area was $1000 \mathrm{~m}$ by $1000 \mathrm{~m}$. The node number was between 10 and 50 nodes. The movement speed of nodes was between 0 and $18 \mathrm{~m} / \mathrm{s}$, and times of simulations were 180 seconds.

To define difference between VNSIP behavior using MCAC and VNSIP behavior without using MCAC, we achieved many types of simulations, and we analyzed behaviors when node speeds and node numbers are increased.

Figures 8 and 9 illustrate bandwidth consumption when establishing SIP communications according respectively to number and mobility of nodes.

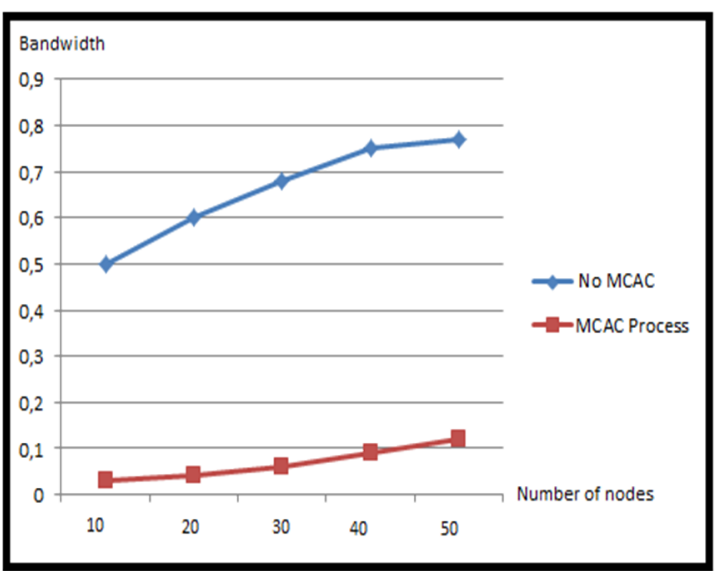

Fig. 8. Bandwidth consumption by number of nodes

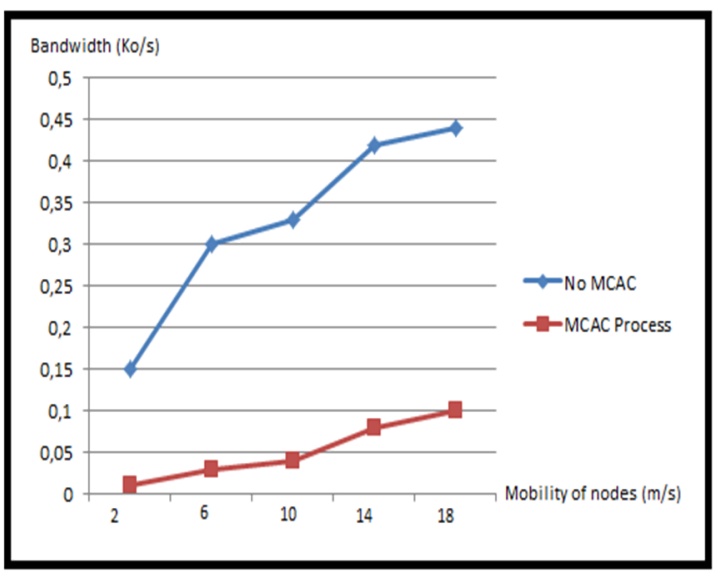

Fig. 9. Bandwidth consumption by mobility of nodes

When we compare both figures, we note that using MCAC process to supervise calls inside the MANET have considerably reduced the consumption of MANET bandwidth; which directly involves improvement of QoS. In fact even if the number of call attempts increases, the MCAC process keeps the number of simultaneous calls in line with the available bandwidth inside the MANET, which guarantees a good QoS for current calls.

\section{CONCLUSION}

This paper proposes a new algorithm to improve the QoS of calls in MANET. We have named this technique, MCAC (MANET Call Admission Control). It aims to ensure QoS by rejecting simultaneous calls in a MANET, when their number reaches a predefined threshold.

We have used this technique to enhance performances of VNSIP approach which suffers from high bandwidth consumption. Simulations has shown that MCAC have considerably reduces bandwidth consumption in MANET, thanks to controlling number of simultaneous calls, which enables to adapt this number to the MANET , which involves to guarantee a good QoS of SIP calls.

\section{REFERENCES}

[1] J. Westcott et G. Lauer, 'Hierarchical routing for very large networks', Proc. IEEE MILCOM '84, pp 214-218, 21-24 October 1984.

[2] J. Rosenberg, H. Schulzrinne, G. Camarillo, A. Johnston, J. Peterson, R. Sparks, M. Handeley et E. Schooler, "SIP: Session Initiation Protocol”, RFC 3261, Juin 2002.

[3] S. Corson, J. Macker, 'Mobile Ad hoc Networking (MANET): Routing Protocol Performance Issues and Evaluation Considerations', REC 2501, Janvier 1999.

[4] M. Jiang, J. Li and Y. C. Tay, "Cluster Based Routing Protocol (CBRP)," IETF Internet Draft draft-ietf-manet-cbrp-spec-01.txt, August 1999.

[5] C. R. Lin and M. Gerla, "Adaptive clustering for mobile, wireless networks," Journal on Selected Areas of Communication, Vol. 15, No. 7, 1997.

[6] J. Rosenberg and H. Schulzrinne, "SIP: Locating SIP Servers", IETF RFC 3263, June 2002.

[7] C. Perkins, E. Belding-Royer, and S. Das “ Ad hoc On-Demand Distance Vector (AODV) Routing ”, IETF RFC 3561, July 2003. 
[8] "The network simulator", available at http://www.isi.edu/nsnam/ns

[9] N. Banerjee, A. Acharya and S.K. Das, "Enabling SIP-Based Sessions in Ad Hoc Networks “, Winet, April 2006.

[10] A.B. Roach, "Session Initiation Protocol (SIP) - Specific Event Notification”, RFC 3265, Juin 2002.

[11]P. Stuedi, M. Bihr, A. Remund et G. Alonso, « Siphoc : Efficient sip middleware for ad hoc networks » studi2007siphoc, LECTURE NOTES IN COMPUTER SCIENCE, 2007, Springer

[12] S. Leggio, J. Manner, A. Hulkkonen, et K. Raatikainen, "Session initiation protocol deployment in ad hoc networks: a decentralized approach", In 2nd International Workshop on Wireless Ad-hoc Networks (IWWAN), London, May, 2005.

[13]I. Mourtaji, M Bouhorma, M. Benahmen, A Bouhdir "A new technique for adapting SIP protocol to Ad hoc Networks: VNSIP (Virtual Network for SIP) Illustration and Evaluation of performance" IJCNCS Volume 1, Issue 1, June 2013. 